\title{
What to expect from ACE inhibitors after myocardial infarction
}

\author{
S G Ball, A S Hall
}

Both doctors and their patients often have unrealistic expectations of treatment. In cardiovascular disease drug treatment is rarely curative, and even when a causal relation exists between a factor and disease, its removal can at best be expected to eliminate only the risk associated with that one factor. Moreover, treatment may not be without adverse as well as beneficial effect. The CAST study offers a salutory lesson that treating patients with little predicted benefit can cause harm.

Medical resource is necessarily limited, and studies of compliance indicate the reluctance of asymptomatic patients to adhere to treatment. Since most patients after myocardial infarction make an excellent recovery, ${ }^{2}$ to target treatment for maximum benefit and minimal harm is attractive. However, selection of high risk patients after myocardial infarction is fraught with difficulty and may be costly. The alternative, treating all for the benefit of a few, has many advocates. Against this background it is useful to examine the role of ACE inhibitors, which now join $\beta$ blockers, thrombolytic agents, and aspirin as one of the most intensively studied groups of drugs in patients after myocardial infarction.

\section{Mortality studies using ACE inhibitors in patients after myocardial infarction} It is important to distinguish postmyocardial infarction studies from studies of chronic left ventricular dysfunction (table 1). CON-
SENSUS $\mathrm{I}^{3}{ }^{3} \mathrm{~V}-\mathrm{HeFT} \mathrm{II}^{4}$ and SOLVD $^{5} 6$ provide important information on long term treatment of patients with chronic impairment of ventricular function. In these studies ischaemic heart disease was the predominant underlying cause of left ventricular dysfunction but, on average, patients were entered many months to years after myocardial infarction.

\section{Postmyocardial infarction studies}

At least seven studies address the impact of ACE inhibitors on the mortality of patients after myocardial infarction ${ }^{7}$ (table 1). ${ }^{7-12}$ Examination of this table shows the difficulty for physicians faced with numerous trials, identified by a myriad of acronyms, to apply appropriately the findings from such studies to their everyday practice. In addition, frequent additional analyses may add to their uncertainty. ${ }^{13}$ We would like to make clear what is known and can be securely put into practice, distinguishing fact from opinion and extrapolation when a more guarded approach is appropriate.

\section{Will all patients benefit from treatment with ACE inhibitors after myocardial infarction?}

Four studies, CONSENSUS II, ISIS-4, GISSI-3, and the Chinese captopril study, have tested a strategy of essentially treating

Table 1 Mortality studies with ACE inhibitors

\begin{tabular}{|c|c|c|c|c|c|c|c|}
\hline Study & $\begin{array}{l}\text { No of } \\
\text { patients }\end{array}$ & Drug used & Time of initiation ${ }^{\star}$ & Follow up & Mortality (\%) & $\begin{array}{l}\text { Outcome (\% risk reduction } \\
(95 \% \text { confidence interval)) }\end{array}$ & $P$ value \\
\hline SAVE $^{7}$ & 2231 & Captopril & $\begin{array}{r}\text { After myocardic } \\
\text { 3-16 days; average } 11 \text { days }\end{array}$ & $\begin{array}{l}\text { al infarction } \\
42 \text { months }\end{array}$ & \multirow{7}{*}{ 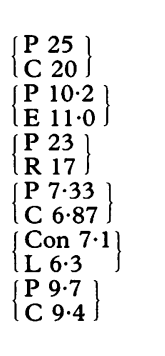 } & $19(3$ to 32$)$ & 0.019 \\
\hline CONSENSUS II & 6090 & Enalapril & Immediately; average $15 \mathrm{~h}$ & 6 months & & $\begin{array}{l}10(7 \% \text { reduction } \\
\text { to } 29 \% \text { increase) }\end{array}$ & 0.26 \\
\hline AIRE $^{9}$ & 2006 & Ramipril & 3-10 days; average 5 days & $\begin{array}{l}\text { Average } 15 \text { months; } \\
\text { minimum } 6 \text { months }\end{array}$ & & $27(11$ to 40$)$ & 0.002 \\
\hline ISIS $-4^{10}$ & 54824 & Captopril & $<24$ hours & 35 days & & $6(<1-12)$ & 0.04 \\
\hline GISSI-3" & 18985 & Lisinopril & $<24$ hours & 6 weeks & & $11(1-20)$ & 0.03 \\
\hline Chinese Captopril Study ${ }^{12}$ & 11345 & Captopril & $<36$ hours & 28 days & & - & NS \\
\hline TRACE $^{12}$ & Ongoing & & & & & & \\
\hline CONSENSUS I $\mathrm{I}^{3}$ & 253 & Enalapril & \multicolumn{2}{|c|}{$\begin{array}{l}\text { Chronic left ventricular dysfunction } \\
>2 \text { months } \\
6 \text { months }\end{array}$} & \multirow{2}{*}{$\begin{array}{l}\left\{\begin{array}{ll}P & 44 \\
E & 26\end{array}\right\} \\
\left\{\begin{array}{ll}\mathrm{H} / \mathrm{I} & 25 \\
\mathrm{E} & 18\end{array}\right\}\end{array}$} & $40(29$ to 50$)$ & 0.002 \\
\hline V-HeFT II ${ }^{4}$ & 804 & Enalapril & $>3$ months & 2 years $\dagger$ & & - & 0.08 \\
\hline $\begin{array}{l}\text { SOLVD: } \\
\text { Treatment }\end{array}$ & 2569 & Enalapril & $>1$ month & $41 \cdot 4$ months & \multirow{2}{*}{ 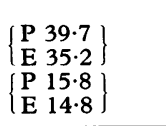 } & $16(5$ to 26$)$ & 0.0036 \\
\hline Prevention $^{6}$ & 4228 & Enalapril & $>1$ month & $37 \cdot 4$ months & & $\begin{array}{l}8(8 \% \text { increase to } \\
21 \% \text { reduction })\end{array}$ & $0 \cdot 30$ \\
\hline
\end{tabular}

P, placebo; Con, control group (not placebo controlled); C, captopril; E, enalapril; R, ramipril; L, lisinopril; H/I, hydralazine-isosorbide dinitrate.

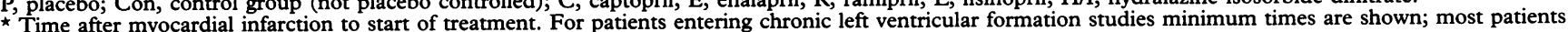
were randomly allocated treatment many months after infarction.

t Time to primary end point. 
"all patients" after myocardial infarction with an ACE inhibitor. Even in these, however, some selection has been imposed, particularly in excluding patients with low blood pressure.

CONSENSUS II offers the most overtly unselected population of patients with presumed myocardial infarction. They were given placebo or intravenous enalaprilat within hours of their myocardial infarction immediately after completion of thrombolysis, followed by oral enalapril. On average, treatment began 15 hours after the onset of chest pain. The ethics review committee stopped the study after recruiting approximately 6000 of the intended 9000 patients for reasons of "statistical futility." The trial if continued would have been unlikely to show the drug statistically superior to placebo. Of concern was that deaths were more common in those taking the drug than in those taking placebo, with some suggestion of particular harm to elderly women. This study, carried out to the high standards associated with large scale Scandinavian trials, adds importantly to our understanding of the use of ACE inhibitors after myocardial infarction, even acknowledging the limitations imposed on interpretation by its premature close. There is no support from this study that immediate ACE inhibition benefits all patients. Indeed, the study highlights the heterogeneity of patients immediately after a myocardial infarction. The clinical course of many patients in the early hours after infarction is unpredictable, and ACE inhibition has the potential to produce harm as well as benefit.

ISIS-4 and GISSI-3 based their protocols on the same premise as CONSENSUS II-that is, the notion that ACE inhibition would have a beneficial effect on the "remodelling" process after myocardial infarction. "Infarct expansion," 14 argued to be the predominant factor underlying future adverse remodelling, ${ }^{15}$ occurs early, within hours of infarction, so that early administration was considered essential. Appreciable ventricular dilatation may ensue in some patients, particularly in the first few weeks after myocardial infarction, and prescription of the drug for 4 to 6 weeks was considered to be a sufficient period of treatment. Captopril or lisinopril were given therefore within 24 hours, but the precise timing was open to clinical judgment, and initial doses were low. Most subjects received the ACE inhibitor more than six hours after the onset of symptoms. Captopril is rapidly absorbed within 30-60 minutes, and even small doses will inhibit ACE activity but the effects may be shortlived. By comparison, lisinopril is absorbed slowly and low doses may take 6-8 hours or more to achieve substantial ACE inhibition, which would then be more sustained than after a low dose of captopril. Less extreme and perhaps later onset ACE inhibition would be predicted from these studies compared with CONSENSUS II. Importantly, many patients with ventricular damage manifesting as low blood pressure, some hours after, but not apparent at the time of the initial event, would not have received treatment in ISIS-4 and GISSI-3. Finally, the findings of CONSENSUS II were presented and later published during the recruitment for ISIS-4 and GISSI-3, which may have influenced selection.

The preliminary findings of the ISIS- 4 and GISSI- 3 trials were reported at the American Heart Association's meeting in Atlanta (November 1993) (table 1). Initial data from both studies showed that mortality was reduced but the effect was small and even with data on nearly 60000 patients in ISIS-4 and 20000 in GISSI-3, significance values were only just within the conventional $5 \%$ levels. At the same meeting the preliminary Chinese captopril study findings (table 1) were reported and even after studying 10000 patients no significant benefit of captopril was apparent. When all three trials are combined, however, a more statistically reassuring but numerically small benefit emerges. Treating 1000 patients prevented about five deaths.

Before individual clinicians blindly accept that they will give ACE inhibitors to all their patients for this small benefit, or alternatively reject the idea of a treatment with such small benefit against cost and inconvenience to the patient, the definitive findings of two other published major studies should be considered.

\section{SAVE STUDY}

In the SAVE study patients were carefully selected on the basis of a reduced radionuclide ejection fraction $(<40 \%)$, and they had no clinical signs or symptoms of heart failure at the time of randomisation. Before randomisation all patients had a test dose of captopril and an exercise test for ischaemia. Some $25 \%$ of patients underwent angioplasty or coronary artery bypass surgery before randomisation into the trial. Treatment with captopril was started on average 11 days after myocardial infarction and follow up was for an average of 42 months. The risk reduction in total mortality was $19 \%$ (95\% confidence interval $3 \%$ to $32 \% ; P=0.019$ ), but no effect was apparent until almost one year into treatment.

\section{AIRE STUDY}

Some 2000 patients were randomly allocated to treatment with placebo or the long acting ACE inhibitor ramipril in addition to usual treatment. Mortality from all causes was the primary end point. Follow up was for a minimum of six months and an average of 15 months. Treatment was started between day 3 and day 10 after myocardial infarction (day 1) and on average at about the fifth day. Patients were selected if they had definite evidence on standard electrocardiographic and enzymatic criteria of myocardial infarction and had clinical evidence of heart failure at some time after admission to hospital. The failure, indicated by a third heart sound with tachycardia, rales at the bases on auscultation, or chest radiology, may have been only transient but would usually have required 
treatment at some time with a diuretic or vasodilator. Patients with severe heart failure were excluded.

In the AIRE study there was a $27 \%(11 \%$ to $40 \%$ ) risk reduction in mortality from all causes in patients receiving ramipril. The finding was highly significant on a log rank test $(2 \mathrm{P}=0.002)$. An important observation was that the mortality curves separated early. Although not part of the primary analysis, a prespecified analysis of early mortality showed a $29 \%$ risk reduction in mortality $(2 \mathrm{P}=0.053)$ even by 30 days. A second analysis on mortality between 30 days and study close, biasing the findings very much against the drug, suggested a further ongoing benefit from ramipril beyond the effects of the first month of treatment. Ramipril prevented both sudden (presumed arrhythmic) death and death associated with progression to severe or resistant heart failure. Importantly, ramipril's effects were independent of thrombolysis or the use of aspirin, men and women gaining similar benefit. Treatment of 1000 patients for one year prevented some 40 deaths.

A case for selecting patients at high risk? Patients after a myocardial infarction do not form a homogeneous group. Most will make a good recovery and by six months to one year will have a prognosis similar to any comparable age group of patients with known ischaemic heart disease. ${ }^{16}$ One substantial readily identifiable group of high risk patients are those who manifest some clinical evidence of heart failure after a myocardial infarction. A recent study undertaken since the introduction of thrombolysis showed that the mortality in patients with evidence of heart failuredenoted by breathlessness, a third heart sound, crackles at the bases, and a need for a diuretic-was extremely high at $46 \%$ in the first year. In many of these patients thrombolysis had probably been unsuccessful, large infarcts ensued, and death occurred within the first 48-72 hours. At the other extreme some would have had only transient failure with minimal damage to their ventricles. The prognosis overall of patients who manifest some clinical evidence of heart failure, even if transient, but survive the first few days after a myocardial infarction is $20-25 \%$ in the first year. The AIRE study showed that physicians using everyday clinical criteria could identify these patients and that treatment with the ACE inhibitor ramipril brought substantial benefit.

Another major predictor of outcome after myocardial infarction is left ventricular ejection fraction. ${ }^{17}$ Whereas clinical criteria

Table 2 Risk stratification after myocardial infarction. Relation between clinical signs and ejection fraction

\begin{tabular}{ll}
\hline Factor & $\begin{array}{l}\text { Relative risk } \\
(95 \% \text { confidence interval) }\end{array}$ \\
\hline Rales > bibasilar & $\begin{array}{l}3.3(2 \cdot 1 \text { to } 5 \cdot 2) \\
\text { Ejection fraction } \leqslant 40 \%\end{array}$ \\
Both & $2 \cdot 4(1.5$ to $3 \cdot 7)$ \\
\hline
\end{tabular}

may be more powerful than ejection fraction in predicting outcome (table 2), the two together select a group at even higher risk. ${ }^{18}$ For any given ejection fraction the observation of heart failure on clinical examination or chest radiology more than doubles subsequent mortality at one year. ${ }^{19} 20$ It is important to recognise, however, that reduced ejection fraction and clinical symptoms identify different but overlapping populations. ${ }^{21}$

Neither ejection fraction nor symptoms were used in the random allocation of patients to the arms of the GISSI- 3 or ISIS- 4 studies, but some information was recorded on these aspects. Subgroup analysis is, however, fraught with difficulties. Thus, for example, in GISSI-3 only women had a significant reduction in mortality with lisinopril, yet no benefit accrued to women in SAVE. In GISSI-3 some three quarters of patients during their stay in a coronary care unit never showed clinical evidence of failure and had an extremely low mortality at six weeks. There was little evidence of benefit from lisinopril in these patients. For those who had some clinical evidence of failure overall mortality in the combined lisinopril treated and control patients was substantially higher. In those manifesting clinical failure the reduction in mortality was substantial. A similar pattern was recorded in those with and without overt failure before randomisation in ISIS-4.

It would be inappropriate to conclude that only patients with signs and symptoms of heart failure can benefit from ACE inhibitors. Indeed, among those without apparent clinical failure some $20-25 \%$ would be expected to have low ejection fractions and the potential for benefit. However, such benefit might not necessarily be expected to be apparent with short term treatment and follow up, as indicated by the observations in SAVE and the chronic left ventricular dysfunction studies (SOLVD treatment/prevention and V-HeFT II). The AIRE and SAVE findings indicate that clinicians using clinical acumen and other assessment of left ventricular dysfunction can identify those most likely to gain from treatment. Importantly, careful selection may protect some from harm.

\section{Timing of treatment with ACE} inhibition after myocardial infarction

An important clinical issue is the timing of the start of treatment. After the findings of CONSENSUS II it is unlikely that much enthusiasm will be found for the use of an intravenous ACE inhibitor. Yet the confidence limits of this study cross those of both ISIS-4 and GISSI-3. Furthermore, as oral captopril is rapidly absorbed, the gain in onset of effect by intravenous compared with oral administration is only 45 minutes to one hour. Many patients in ISIS-4 received captopril within six hours of the onset of their chest pain against an average time of 15 hours for intravenous enalaprilat in CONSENSUS II. Although there may have been considerable overlap in timing of first achieving ACE inhibition, 
the intravenous and oral regimen in CONSENSUS II, given the different characteristics of long acting enalapril compared with short acting captopril, would almost certainly have produced longer lasting and more profound early ACE inhibition. In the AIRE study benefit was apparent whether patients were randomised before or after the fifth day, and patients in SAVE did not receive treatment until an average of 11 days after infarction. However, in the AIRE study even by the time of discharge from hospital more patients had died in the placebo group than in the ramipril group. In both these studies patients were clinically stable. Whereas early treatment seems desirable, delay in administration beyond the first day makes assessment of clinical stability and identification of patients most likely to benefit more certain and would seem prudent.

\section{Can patients expect to gain above conventional treatment?}

The importance of early thrombolysis and aspirin treatment is beyond question, though even here some have much more to gain than others; a few will be harmed. Though the evidence for benefit from $\beta$ blockers was all gained before the introduction of thrombolysis, it would be perverse to eschew their use. Intravenous $\beta$ blockade has achieved little favour for use in the United Kingdom, yet the potential for lives saved per 1000 patients treated in the first ISIS trial was almost twice that seen with captopril in the most recent ISIS study. Recognition of the heterogeneity of the patients seems essential for sensible practice. At one end of the range $\beta$ blockers seem obligatory, but when left ventricular function is obviously impaired their use may be inappropriate. For ACE inhibitors the converse situation obtains, with benefit beyond doubt for those with poor ventricular function after surviving the unstable first few hours after infarction. Between these extremes lie a broad group of patients who should benefit from both drugs, but probably to differing degrees. The currently published evidence from the SAVE and AIRE trials indicates that the benefit of ACE inhibition is additive to that of thrombolysis, aspirin, $\beta$ blockade, and diuretics.

\section{ACE inhibitors as prophylactic treatment?}

So far we have concentrated on the effects of ACE inhibitors on mortality since this is an end point of which there can be no doubt. However, a persuasive argument can be made that these drugs may alter the natural history of coronary artery disease and left ventricular dysfunction. Data are limited. ${ }^{6} 722$ Even short term treatment at a critical time may lead to long term benefit, as seen with streptokinase given after myocardial infarction. Subtle but, nevertheless, worthwhile survival benefit from treatment might not be expected to be apparent for some years. This aspect is illustrated in the prevention arm of the SOLVD study. ${ }^{6}$ Long term treatment with enalapril in "asymptomatic" patients with evidence of impaired left ventricular function did not significantly reduce mortality but decreased hospital admissions for heart failure. However, a caveat for any trial of this design is that it cannot distinguish true prevention from the effects of giving an active agent before the onset of symptoms, thereby merely treating in advance of actual need.

In the SAVE study showed without signs or symptoms of heart failure at randomisation who had impaired left ventricular function (indicated by a reduced radionuclide ejection fraction) benefited not only from longer survival but also from preservation of ventricular function as assessed by measurement of ejection fraction. Similarly, in the AIRE study patients with signs and symptoms of ventricular dysfunction, even if transient, lived longer and fewer exhibited deterioration of ventricular function assessed clinically. The observations of these two studies and the SOLVD prevention trial support a role for the ACE inhibitors in preventing deterioration of ventricular function. More difficult for the clinician is to decide the degree of left ventricular dysfunction which justifies treatment and how to assess that dysfunction reliably.

The potential for preventing reinfarction adds a further dimension. In the SAVE study patients receiving captopril had fewer myocardial infarctions as defined by the investigators than had those receiving placebo. The effect was less apparent for reinfarctions defined by the end points committee, and the effect disappeared if "silent reinfarctions" were included..$^{23}$ The AIRE study was shorter, it had less than half the number of end point committee validated reinfarctions observed in the SAVE study, and only a marginal trend to fewer reinfarctions was seen in the group given ACE inhibitors. Furthermore, in the SAVE and AIRE studies patients who had a second infarction after the index infarction permitting entry into the trial had twice the mortality of patients without reinfarctions, but similar benefit from ACE inhibitors was seen in both groups in both studies. The preliminary findings of GISSI-3 and ISIS- 4 with more than 2000 reinfarctions show no benefit from ACE inhibition, but the treatment was for a maximum of six weeks. Studies which prospectively address prevention of myocardial infarction in a wider group of patients to include those with apparently normal ventricular function are required to resolve this important issue.

\section{Conclusion}

A substantial proportion of patients after myocardial infarction can expect considerable benefit from treatment with ACE inhibitors. Patients with the combination of clinical signs and symptoms of heart failure and low ejection fractions have most to gain and treatment should not be unnecessarily delayed. 
Currently, prescriptions of diuretics for heart failure in the United Kingdom are estimated to be 10 times higher than those of ACE inhibitors. Doctors should ensure that the majority of these patients and those who are prescribed diuretics receive appropriate treatment with an ACE inhibitor, rather than be preoccupied with debating the marginal benefits.

1 CAST Investigators. Preliminary report: effect of encainide and flecainide on mortality in a randomized trial of and flecainide on mortality in a randomized trial of arrhythmia suppression after
Engl $\boldsymbol{f}$ Med 1989;321:406-12.

2 Stevenson $R$, Ranjadaylan $K$, Wilkinson $P$, Roberts $R$, Timmis AD. Short and long term prognosis of acute myocardial infarction since introduction of thrombolysis. myocardial infarction since

3 CONSENSUS Trial Study Group. Effects of enalapril on mortality in severe congestive heart failure; results of the cooperative north Scandinavian enalapril survival study (CONSENSUS). $N$ Engl f Med 1987;316:1429-35.

4 Cohn JN, Johnson G, Ziesche S, et al. A comparison of enalapril with hydralazine-isosorbide dinitrate in the treatment of chronic congestive heart failure. $N$ Engl $f$ Med 1991;325:303-10.

5 SOLVD Investigators. Effect of enalapril on survival in patients with reduced ejection fractions and congestive heart failure. N Engl f Med 1991;325:293-302.

6 SOLVD Investigators. Effect of enalapril on mortality and the development of heart failure in asymptomatic patients with reduced left ventricular ejection fractions. $N$ Engl f Med 1992;327:685-91.

7 Pfeffer MA, Braunwald E, Moye LA, et al. Effect of captopril on mortality and morbidity in patients with left captopril on mortality and morbidity in patients with left ventricular dysfunction after

8 Swedburg K, Held P, Kjekshus J, et al on behalf of the CONSENSUS II Study Group. Effects of early administration of enalapril on mortality in patients with administration of enalapril on mortality in patients with acute myocardial infarction. Results of the cooperative
north Scandinavian enalapril survival study north Scandinavian enalapril survival study
(CONSENSUS II). $N$ Engl $₹$ Med 1992;327:678-84

9 Acute Infarction Ramipril Efficacy (AIRE) Investigators.
Effects of ramipril on mortality and morbidity of survivors of acute myocardial infarction with clinical evidence of heart failure. Lancet 1993;342:821-8.

10 ISIS-4 Collaborative Group. Fourth international study of infarct survival: protocol for a large simple study of the effects of oral mononitrate, or oral captopril, and of intravenous magnesium. Am $\mathcal{f}$ Cardiol 1991;68 (suppl):87-100D

11 GISSI-3. Study protocol on the effects of lisinopril, of nitrates, and of their association in patients with acute myocardial infarction. Am f Cardiol 1992;70:62-9C.

12 Hall AS, Tan LB, Ball SG. Inhibition of ACE/kinase II, acute myocardial infarction and survival. Cardiovasc Res 1994;28:199-200

13 Mills JL. Data torturing. N Engl f Med 1993;329:1196-9.

14 Hutchins GM, Bulkley BH Infarct expansion versus extension: two different complications of acute myor

5 Pfeffer MA, Braunwald E. Ventricular remodeling after myocardial infarction. Circulation 1990;81:1161-72.

16 Dwyer EM, Greenberg H, Case RB, Multicenter Postinfarction Research Group. Association between transient pulmonary congestion during acute myocardial transient pulmonary congestion during acute myocardial infarction and high incidence

17 White HD, Norris RM, Brown MA, Brandt PWT, Whitlock RML, Wild CJ. Left ventricular end-systolic Whitlock RML, Wild CJ. Left ventricular end-systolic
volume as the major determinant of survival after volume as the major determinant of survival after recovery from

8 Multicenter Postinfarction Research Group. Risk stratification and survival after acute myocardia infarction. N Engl f Med 1983;309:331-6.

19 Gottlieb S, Moss AJ, McDermott M, Eberly S Interrelation of left ventricular ejection fraction pulmonary congestion and outcome in acute myocardial infarction. Am f Cardiol 1992;69:977-84.

20 Nicod P, Gilpin E, Dittrich $\mathrm{H}$, et al. Influence on prognosis and morbidity of left ventricular ejection fraction with and without signs of left ventricular failure after with and without signs of left ventricular failure after acute

21 Ball SG, Hall AS, Murray GD. ACE-inhibition atherosclerosis and myocardial infarction-the AIRE atherosclerosis and myocardial infarction-the AlR

22 Yusuf S, Pepine CJ, Garces C, et al. Effect of enalapril on myocardial infarction and unstable angina in patients myocardial infarction and unstable angina in patients

23 Ganley CJ, Hung HMJ, Temple R. More on the survival and ventricular enlargement trial. $N$ Engl $f \mathrm{Med}$ and ventricular 